Original Research Articles

\title{
Diagnostic Value of Vascular Endothelial Growth Factor C (VEGF-C) and CA 15-3 in Breast Cancer
}

\author{
Ajeng Kurnia Wardhani ${ }^{1 *}$, Indranila Kustarini Samsuria ${ }^{2}$, Meita Hendrianingtyas ${ }^{2,3}$, Edward Kurnia \\ Setiawan Limijadi ${ }^{2}$, Ria Triwardhani ${ }^{2,4}$ \\ ${ }^{1}$ Faculty of Medicine, Universitas Diponegoro, Semarang, Indonesia \\ ${ }^{2}$ Clinical Pathology Departement, Faculty of Medicine, Universitas Diponegoro, Semarang, Indonesia \\ ${ }^{3}$ Diponegoro National Hospital Semarang, Indonesia \\ ${ }^{4} d r$. Kariadi Hospital, Semarang, Indonesia
}

\section{Article Info}

History

Received: 07 July 2021

Accepted: 17 Dec 2021

Available: 31 Dec 2021

\begin{abstract}
Background: Expression of VEGF-C and CA 15-3 may be useful to differentiate between malignant and benign breast tumour because VEGF-C plays a role in promoting angiogenesis and lymphangiogenesis in malignant processes and CA 15-3 is the soluble form of transmembrane protein MUC1, a tumour marker which shows higher expression in breast cancer.

Objective: To determine the diagnostic value of VEGF-C and CA 15-3 as blood-based tumour markers in patients with breast cancer should the more invasive pathology examination is unavailable or undesirable.

Methods: This diagnostic cohort study recruited 76 patients that underwent surgical biopsy procedures at Dr. Kariadi and Pantiwilasa Citarum Hospitals Semarang. The VEGF-C and CA 15-3 levels in blood specimens taken before surgical biopsy procedure was determined using ELISA method. An ROC curve and AUC were used to establish the cut-off points and diagnostic value. Pathology examination results from the biopsy specimens were used as the gold standard.

Results: The cut-off value for VEGF-C and CA $15-3$ were $989.50 \mathrm{pg} / \mathrm{mL}$ and 74.00 U/mL. Sensitivity for VEGF-C, CA 15-3 and VEGF-C+CA 15-3 were 76.6\%, 64.1\% and $89.1 \%$. Specificity for VEGF-C, CA 15-3 and VEGF-C+CA 15-3 were 75.0\%, $75.0 \%$ and $50.0 \%$. The AUC for VEGF-C, CA 15-3 and VEGF-C+CA 15-3 was 0.831 $(95 \% \mathrm{CI}=0.727-0.934), 0.742(95 \% \mathrm{CI}=0.628-0.856)$ and $0.840(95 \% \mathrm{CI}=0.742-$ 0.938 ).

Conclusion: VEGF-C in combination with CA 15-3 performed better as diagnostic parameter and has better accuracy as a tumour marker for breast cancer in comparison with each marker alone.
\end{abstract}

Keywords: Breast cancer; VEGF-C; CA 15-3; tumours marker; diagnostic value. Permalink/ DOI: https://doi.org/10.14710/jbtr.v7i3.11681

\section{INTRODUCTION}

Breast cancer is the most common type of cancer in women, with a prevalence of $13.2 \%$ in South-East Asia by the end of 2020, and is also reported as one of the leading causes of cancer-related mortality. ${ }^{1-4}$ Early and accurate diagnosis was reported to help improve prognosis and increase patient life expectancy. ${ }^{4,5}$ Starting therapy at an earlier stage of the disease is expected to help cut treatment costs. Cancer cells in early-stage disease also generally show lower mutational complexity and clonal heterogeneity and therefore have a lower risk of developing resistance to therapy. ${ }^{6}$ Therefore, early detection and screening are considered to play an important role in the management of breast cancer. ${ }^{7}$

\footnotetext{
* Corresponding author:

E-mail: ajeng3005@gmail.com

(Ajeng Kurnia Wardhani)
} 
Histopathological examination of biopsy specimens is the gold standard for establishing a diagnosis in patients with breast tumours, ${ }^{8}$ although this method is known to have several shortcomings, such as causing pain and discomfort, invasive in nature, and the results can be affected by erroneous sampling process, the biopsy specimen, as well as the level of expertise and experience of the examiner. Surgical biopsy is also relatively undesirable in patients taking anticoagulant, patients with overlying skin infections, and during pregnancy or breast-feeding. ${ }^{6,9}$ This shows the need for a more convenient non-invasive screening method to do early detection of breast cancer cases in a relatively accurate manner.

Expression of serum tumour markers is a non-invasive screening methods that can detect malignant cell transformation since early stage of breast cancer. ${ }^{7,10}$ Expression of tumour markers such as VEGF-C and CA 15-3 may be useful to differentiate between malignant and benign breast tumour because VEGF-C plays a role in promoting angiogenesis and lymphangiogenesis in malignant processes and CA 15-3 is the soluble form of transmembrane protein MUC1, a tumour marker which shows higher expression in breast cancer. The use of these diagnostic tumour marker is also considered quite promising because the diagnosis can be made without being influenced by the level of expertise and experience of the examiner or erroneous sampling process that we may encounter in conventional histopathological examination. ${ }^{11}$

CA 15-3 examination was reported to have a relatively low sensitivity in stage I and II breast cancer, and was more widely used as an early indicator of cancer recurrence, the presence of residual disease, and to evaluate remission or to predict prognosis. ${ }^{7,12,13}$ Previous studies have also generally investigated VEGF-C as a prognostic marker, as a marker of metastases, or to predict life expectancy, ${ }^{3,14}$ and only one study in Poland had previously evaluated the diagnostic value of these two tumour markers. ${ }^{10}$ The current study aimed to evaluate the accuracy and reproducibility of VEGF-C and CA 15-3 as a blood-based diagnostic test for breast cancer should the more invasive pathology examination is unavailable or undesirable.The results of this study were expected to help develop a new panel of tumour markers that can be used in early diagnosis and screening for breast cancer in the future.

\section{MATERIALS AND METHODS \\ Study design}

This diagnostic study recruited patients that underwent surgical biopsy procedures at Dr. Kariadi and Pantiwilasa Citarum Hospitals Semarang between May and December 2020. The VEGF-C and CA 15-3 levels in blood specimens taken before surgical biopsy procedure was determined using ELISA with double antibody sandwich technique. Pathology examination results from the biopsy specimens were used as the gold standard.

\section{Study subjects}

Combined sensitivity and specificity value for a diagnostic test was expected to at least be 1.5 , and to be able to recommend the clinical use of such tests, ideally the test should be able to identify significantly more cases correctly (sensitivity $\geq 80 \%$ ) and have very high specificity $(\geq 97 \%) .{ }^{15}$ The sample size in the current study was calculated using the formula for diagnostic test, with expected sample proportion of 0.84 and an absolute precision of $10 \%$. The sample was calculated as a whole without establishing specific calculated numbers of patients having tumors or no tumors to be recruited in order to reject the null hypothesis.

Inclusion criteria for the current study were over 17 years old female patients with no previous history of breast cancer, operable tumour where histopathology examination was conducted, body temperature between $36-37.4^{\circ} \mathrm{C}$, no history of surgery during the last 6 months before biopsy, and normal haemoglobin, leukocytes, or platelet values. Eighty patients that underwent surgerybiopsy for breast tumour had agreed to participate in the study. Four patients were then excluded, 3 because they were found to have recurrent breast cancer and 1 because she already had a biopsy examination in another hospital. Seventy-six patients were selected as study subjects, 64 with malignant and 12 with benign tumour.

\section{Data analysis}

The collected data is processed through editing, tabulation, coding and entry processes using IBM SPSS statistics version 27.0. Receiving Operating Characteristics (ROC) curve and Area Under the ROC Curve (AUC) were used to establish the cut-off points, which was then used to calculate sensitivity, specificity, positive predictive value (PPV), and negative predictive value (NPV) to establish the diagnostic value.

\section{Ethical clearance}

The study had been approved by The Medical Research Ethics Committee at The Faculty of Medicine Diponegoro University/Dr. Kariadi Hospital Medical Center Semarang.

\section{RESULTS}

Seventy-six patients that underwent breast tumour surgery-biopsy in Dr. Kariadi General Hospital Medical Center Semarang were included in the current study. The study subjects were between 19 to 65 years old (mean \pm SD, $48.01 \pm 11.30$ years). Histopathology showed malignant tumour in 64 patients and benign tumour in 12 patients. Patients with malignant histopathology were significantly older and had significantly younger menarche age in comparison to patients with benign histopathology $(\mathrm{p}<0.05)$. The malignant histopathology group showed significantly higher proportion of patients who had entered menopause and had used hormonal contraception for more than 3 years in comparison to those with benign histopathology. The malignant histopathology group also showed higher proportion of patients with a history of breastfeeding for less than 1year, albeit statistically insignificant (Table 1). The current study did not consider the stages or types of breast cancer in the analysis. Consequently, cancer staging was not recorded, and ER, PR, or HER2 status was not evaluated during the study period. 
Table 1. Characteristics of study subjects in malignant and benign histopathology groups

\begin{tabular}{|c|c|c|c|}
\hline \multirow{2}{*}{ Variables } & \multicolumn{2}{|c|}{ Histopathology Results } & \multirow[t]{2}{*}{$p$} \\
\hline & Malignant & Benign & \\
\hline Age (year) (mean \pm SD) & $49.69 \pm 10.71$ & $39.08 \pm 10.51$ & $0.002^{\S *}$ \\
\hline \multicolumn{4}{|l|}{ History of breastfeeding $>1$ year $(n, \%)$} \\
\hline Yes & $5(62.5)$ & $3(37.5)$ & $0.107^{\wedge}$ \\
\hline No & $59(86.8)$ & $9(13.2)$ & \\
\hline \multicolumn{4}{|l|}{ Menopause (n, \%) } \\
\hline Yes & $45(93.8)$ & $3(6.3)$ & $0.007^{*^{*}}$ \\
\hline No & $19(67.9)$ & $9(32.1)$ & \\
\hline \multicolumn{4}{|l|}{ Hormonal contraception $>3$ years $(\mathrm{n}, \%)$} \\
\hline Yes & $56(91.8)$ & $5(8.2)$ & $0.001^{*}$ \\
\hline No & $8(53.3)$ & $7(46.7)$ & \\
\hline Age of menarche (year) (median, IQR) & $11(10-12)$ & $12(11-13)$ & $0.026^{* *}$ \\
\hline VEGF-C $(\mathrm{pg} / \mathrm{ml})($ mean $\pm \mathrm{SD})$ & $1368.86 \pm 1428.09$ & $949.17 \pm 52.25$ & $<0.001 * *$ \\
\hline $\mathrm{CA} 15-3(\mathrm{U} / \mathrm{ml})($ mean $\pm \mathrm{SD})$ & $159.48 \pm 157.67$ & $52.00 \pm 23.59$ & $0.008^{* *}$ \\
\hline
\end{tabular}

*Significant ( $<$ 0,05); ${ }^{\S}$ Independent $t$ test; ${ }^{\star}$ Mann whitney test; ${ }^{\wedge}$ Fisher’s exact test; IQR, interquartile range.

Table 2. AUC and cut-off value for VEGF-C, CA 15-3 and VEGF-C + CA 15-3

\begin{tabular}{lccccc}
\hline \multirow{2}{*}{ Variables } & \multirow{2}{*}{ AUC } & \multirow{2}{*}{} & \multicolumn{2}{c}{$\mathbf{9 5 \%}$ CI } & \multirow{2}{*}{ Cut-off point } \\
\cline { 4 - 5 } & & & Min & Max & \\
\hline VEGF-C & 0.831 & 0.000 & 0.727 & 0.934 & 989.50 \\
CA 15-3 & 0.742 & 0.008 & 0.628 & 0.856 & 74.00 \\
VEGF-C + CA 15-3 & 0.840 & $<0.001$ & 0.742 & 0.938 & - \\
\hline
\end{tabular}

$\mathrm{AUC}=$ area under the ROC curve; $95 \% \mathrm{CI}=95 \%$ confidence interval.

Table 3. Diagnostic value of VEGF-C, CA 15-3, and VEGF-C + CA 15-3

\begin{tabular}{|c|c|c|c|c|c|c|c|c|c|}
\hline \multirow[b]{2}{*}{ Variables } & \multirow[b]{2}{*}{ Category } & \multicolumn{2}{|c|}{ PA Results } & \multirow{2}{*}{$\begin{array}{c}\text { Sens. } \\
(\%)\end{array}$} & \multirow{2}{*}{$\begin{array}{c}\text { Spec. } \\
(\%)\end{array}$} & \multirow{2}{*}{$\begin{array}{l}\text { PPV } \\
(\%)\end{array}$} & \multirow{2}{*}{$\begin{array}{l}\text { NPV } \\
(\%)\end{array}$} & \multirow{2}{*}{$\begin{array}{l}\text { Acc } \\
(\%)\end{array}$} & \multirow[b]{2}{*}{$p$} \\
\hline & & + & - & & & & & & \\
\hline \multirow[t]{2}{*}{ VEGF-C } & $\geq 989.50$ & 49 & 3 & 76.6 & 75.0 & 94.2 & 37.5 & 83.1 & 0.001 \\
\hline & $<989.50$ & 15 & 9 & & & & & & \\
\hline \multirow[t]{2}{*}{ CA $15-3$} & $\geq 74.00$ & 41 & 3 & 64.1 & 75.0 & 93.2 & 28.1 & 74.2 & 0.049 \\
\hline & $<74.00$ & 23 & 9 & & & & & & \\
\hline VEGF-C & $\geq$ cut-off & 57 & 6 & 89.1 & 50.0 & 90.5 & 46.2 & 84.0 & 0.018 \\
\hline CA $15-3$ & $<$ cut-off & 7 & 6 & & & & & & \\
\hline
\end{tabular}

Sens $=$ sensitivity Spec $=$ specificity PPV = positive predictive value; NPV = negative predictive value; Acc $=$ accuracy.

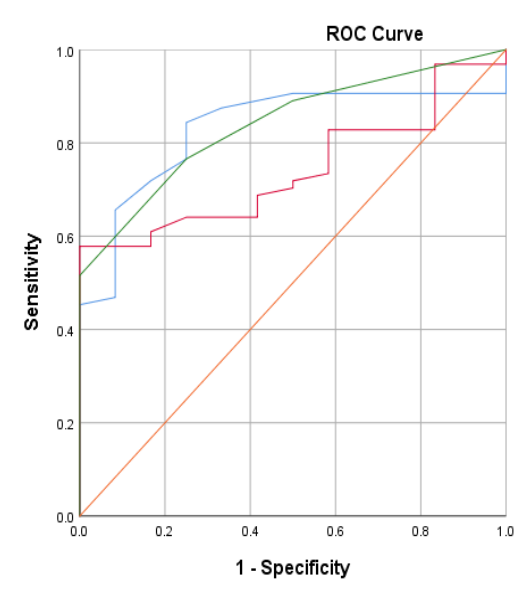

Figure 1. ROC curve for VEGF-C, CA 15-3, and VEGF-C + CA $15-3$

Patients with malignant histopathology showed significantly higher mean VEGF-C levels in comparison to patients with benign histopathology $(1368.86 \pm 1428.09 \mathrm{pg} / \mathrm{mL}$ vs. $949.17 \pm 52,25 \mathrm{pg} / \mathrm{mL}$; $\mathrm{p}<0.05)$. Mean CA 15-3 levels was also significantly higher in patients with malignant in comparison to benign histopathology $(159.48 \pm 157.67 \mathrm{U} / \mathrm{mL} \quad$ vs. 52.00 $\pm 23.59 \mathrm{U} / \mathrm{mL} ; \mathrm{p}<0.05$ ) (Table 1).

Determination of cut-off value for VEGF-C and CA 15-3

Receiver operating characteristic (ROC) curve and area under the ROC curve (AUC) was used to determine the optimal cut-off value for VEGF-C and CA 15-3 based on the histopathology of the tumour. Cut-off value for VEGF-C was $989.50 \mathrm{pg} / \mathrm{mL}$ and for CA 15-3 was 74.00 $\mathrm{U} / \mathrm{mL}$. Tumour markers were considered to show a positive result when the serum levels were found to be equal to or higher than the cut-off value, and were considered to show a negative result when the serum levels were found to be lower than the cut-off value (Table 2, Figure 1).

\section{Diagnostic value of VEGF-C and CA 15-3}

Diagnostic accuracy showed the proportional agreement between the diagnostic test result that was made based on the cut-off value for the studied tumour markers in comparison to the result of the reference test or gold standard. There were several factors that can be considered in determining the accuracy of a diagnostic method, such as sensitivity, specificity, positive predictive value (PPV), and negative predictive value 
(PPV). A number of these factors can be calculated based on the number of true positive results (TP), true negative results (TN), false positive results (FP) and false negative results (FN) with the following formula: ${ }^{15}$
a) $\quad$ Sensitivity $=\mathrm{TP} /(\mathrm{TP}+\mathrm{FN})$
b) Specificity $=\mathrm{TN} /(\mathrm{TN}+\mathrm{FP})$
c) $\mathrm{PPV}=\mathrm{TP} /(\mathrm{TP}+\mathrm{FP})$
d) $\mathrm{NPV}=\mathrm{TN} /(\mathrm{FN}+\mathrm{TN})$

Positive or negative diagnostic test results to ascertain whether the patient has a malignant or benign tumour were determined according to VEGF-C and CA 15-3 cut-off value that has been previously calculated.

Based on the predetermined cut-off value, among 64 patients with malignant histopathology, 49 showed positive results for VEGF-C, resulting in a sensitivity of $76.6 \%$, and among 12 patients with benign histopathology, 9 showed negative results, resulting in a specificity of $75.0 \%$. Forty-one patients with malignant histopathology showed positive results for CA 15-3, resulting in a sensitivity of $64.1 \%$, and 9 patients with benign histopathology showed negative results, resulting in a specificity of $75.0 \%$. Positive results for combined VEGF-C and CA 15-3 were found in 57 patients with malignant histopathology, resulting in a sensitivity of $89.1 \%$, and negative results were found in 6 patients with benign histopathology, resulting in a specificity of $50.0 \%$ (Table 3).

Among 52 patients with positive results for VEGF-C, 49 were found to have malignant histopathology, thus resulting in a PPV of $94.2 \%$, and among 24 patients with negative results, only 9 were found to have benign histopathology, thus resulting in an NPV of $37.5 \%$. Concurrently, among 44 patients with positive results for CA $15-3,41$ were found to have malignant histopathology, thus resulting in a PPV of $93.2 \%$, and among 32 patients with negative results, only 9 were found to have benign histopathology, thus resulting in an NPV of $28.1 \%$. Combined VEGF-C and CA 15-3 showed positive results in 63 patients, where 57 were found to have malignant histopathology, thus resulting in a PPV of $90.5 \%$, and showed negative results in 13 patients, where 6 were found to have benign histopathology, thus resulting in an NPV of $46.2 \%$ (Table 3).

\section{DISCUSSION}

The current study involving 76 subjects with breast tumours between the age of 19 to 70 years have found 64 subjects with breast cancer (malignant histopathology) and 12 with benign breast tumours (benign histopathology). Patients with breast cancer were significantly older, had significantly younger menarche age, and showed higher proportion of patients who had used hormonal contraception for more than 3 years in comparison to patients with benign tumour. Age was one of the most important risk factors for breast cancer, where the incidence was reported to increase significantly with age and reaches its peak in the age of menopause. Younger age of menarche and the use of hormonal contraception has also been reported as a risk factor associated with an increased incidence of breast cancer. $^{16-18}$
Serum tumour markers expression was reportedly useful in early detection of malignant cell transformation, thus may be utilized as a non-invasive screening method in breast tumours. ${ }^{7,10}$ VEGF-C and CA 15-3 are often used as biomarkers in breast cancer, albeit rarely for diagnostic purposes. The current study evaluated the diagnostic value of VEGF-C and CA 15-3 as serum tumour markers in breast cancer. CA 15-3 has a relatively low sensitivity in stage I and II of breast cancer, and was more widely used as an early indicator of cancer recurrence, the presence of residual disease, and to evaluate remission or to predict prognosis..$^{7,12,13}$ Previous studies also mostly investigated VEGF-C as a prognostic marker, as a marker of metastases, or to predict life expectancy. ${ }^{3,14}$ Only one previous study in Poland had evaluated the diagnostic value of these two tumour markers. ${ }^{10}$

Vascular endothelial growth factor C (VEGF-C) was a growth factors that played an important role in the formation of blood vessels in VEGFR-3 and VEGFR-2 receptor-positive breast cancer tissue. Intraductal and invasive breast cancer cells were found to express VEGF$\mathrm{C}$ protein. ${ }^{19}$ Patients with malignant histopathology in the current study showed a significantly higher mean VEGF$\mathrm{C}$ level than patients with benign histopathology. Similarly, previous studies by Zajkowska et al. and Thammineni et al. also found significantly higher VEGF$\mathrm{C}$ expression in patients with breast cancer as opposed to patients with benign breast tumours. ${ }^{7,20}$ The increase in VEGF-C levels was also reported to be positively correlated to the severity of breast cancer assessed by TNM stage. This shows that plasma VEGF-C levels may potentially be utilized to differentiate between breast cancer and benign breast tumours. ${ }^{7}$

Cancer antigen 15-3 or CA 15-3 was one of the most frequently examined tumour markers for breast cancer, both in daily clinical practice and for research purposes. $^{13,21}$ CA 15-3 examination will detect the presence of a soluble form of the protein antigen MUC1, ${ }^{1,22,23}$ a large transmembrane mucin glycoprotein which is known to be over-expressed in patients with breast cancer. ${ }^{22,24}$ Patients with malignant histopathology in the current study showed significantly higher CA 15-3 levels in comparison to patients with benign histopathology. Similarly, previous studies by $\mathrm{Fu}$ et al., Lawicki et al., and $\mathrm{Fu}$ and $\mathrm{Li}$ also found higher serum CA 15-3 levels in breast cancer patients as opposed to patients with benign breast tumours. ${ }^{21,25,26}$ Increased CA 15-3 expression can be found at all stages of breast cancer, and appeared to be positively correlated with the tumour stage. ${ }^{25}$

\section{Diagnostic Value of VEGF-C and CA 15-3}

One of the main criteria for assessing the diagnostic value of a tumour marker is the sensitivity/specificity chart or ROC curve. ${ }^{7}$ The ROC curve was a mapping of sensitivity against 1-specificity for all possible cut-off values between cases and controls. The diagnostic value of a tumour marker was assessed using the area under the ROC curve (AUC) and/or the area under the partial ROC curve (pAUC). A tumour marker with $\mathrm{AUC}=1 \mathrm{can}$ completely differentiates between patients with breast cancer or benign breast tumours. Meanwhile, $\mathrm{AUC}=0.5$ indicates that there was no difference in the distribution 
of tumour marker values between the two groups. ${ }^{27}$

Diagnostic value based on the AUC will show the total accuracy of a tumour marker, where a tumour marker is considered to have better performance if the ROC curve was closer to the upper left corner of the graph, thus indicating an AUC value that is closer to 1.0 (representing a combination of perfect or $100 \%$ sensitivity and specificity value). An AUC value of 0.7 to 0.8 indicates a moderate diagnostic value, 0.8 to 0.9 indicates a good diagnostic value, and more than 0.9 indicates a very good diagnostic value. An AUC value of 0.8 and above is usually acceptable to clinicians. ${ }^{7,28,29}$ The current study obtained AUC values of 0.831 for VEGF-C, 0.742 for CA 15-3, and 0.840 for combined VEGF-C + CA 15-3, so it can be concluded that CA 153 has moderate diagnostic value while VEGF-C and combined VEGF-C + CA 15-3 has good diagnostic value.

Previous study by Zajkowska et al. has compared several tumour markers in breast cancer and found similar results, with relatively high AUC values for VEGF-C (0.767), CA 15-3 (0.757), and combined VEGF-C + CA 15-3 (0.847). The results of this study indicated that VEGF-C and CA 15-3 were good candidates to help establish the diagnosis of breast cancer, where the use of combined VEGF-C + CA 15-3 appears to have a better diagnostic value than the use of each individual markers. ${ }^{7}$ These results were in agreement with the results obtained in the current study, where the combined VEGF-C + CA 15-3 tumour markers showed higher AUC value than VEGF-C and CA 15-3 individually.

\section{Cut-off Value for VEGF-C and CA 15-3}

ROC curve analysis and AUC calculation was used to determine the diagnostic accuracy of VEGF-C and CA 15-3 in breast tumours and to estimate the optimal cutoff value of these tumour markers in the current study. The cut-off value was determined to dichotomize the results of the examination, namely to determine whether the results were positive or negative (malignant or benign tumours). The cut-off value will be considered as optimal if it can be used to classify most of the research subjects correctly. ${ }^{27}$

Analysis to determine the optimal cut-off value requires a combination of the area under the ROC curve (AUC) along with determining the sensitivity and specificity values at the same time. This method defines the optimal cut-off value as the value that minimizes the sum total of the absolute difference values between AUC and sensitivity and AUC and specificity, provided that the difference between sensitivity and specificity values appears to be minimal. ${ }^{27}$ The current study obtained a cut-off value of $989.50 \mathrm{pg} / \mathrm{mL}$ for VEGF-C and 74.00 $\mathrm{U} / \mathrm{mL}$ for CA $15-3$. This cut-off value was relatively vast difference with the ones obtained from previous studies by Zajkowska et al., which were $1552.85 \mathrm{pg} / \mathrm{mL}$ for VEGF-C and $18.45 \mathrm{U} / \mathrm{mL}$ for CA $15-3 .^{7}$ This might be influenced by differences in sample size and the characteristics study subjects. Zajkowska et al. recruited 120 patients with breast cancer between the age of 39-83 years, where $82.5 \%$ have entered menopause, ${ }^{7}$ unlike the current study which only recruited 64 breast cancer patients with a wider age range, between 19-70 years, and with lower percentage of patients who have entered menopause $(70.3 \%)$. In comparison to pre-menopausal breast cancer patients, patients who have entered menopause was reported to have a lower mean VEGF-C expression, ${ }^{30}$ and a higher CA $15-3$ expression. ${ }^{31}$ This difference in proportion of pre- and post-menopausal age patients between the current study and the study by Zajkowska et al. may have a bearing on the difference in VEGF-C and CA 15-3 levels between the two studies. Research from Zajkowska et al. also calculates the cutoff value based on the breast cancer stages, ${ }^{7}$ unlike the current study which only calculates the cut-off value as a whole without considering the stage of the disease.

\section{Diagnostic Accuracy of VEGF-C and CA 15-3 in Breast Cancer}

The accuracy of a diagnostic method can be determined by comparing the results with a reference standard or a diagnostic gold standard method that can determine whether the patient does have the disease with a high level of confidence. The ideal reference standard was generally expected to have a sensitivity and specificity level of $100 \%$, although sometimes it has several limitations, where this method might be more difficult to perform, more expensive, and more invasive. ${ }^{15}$ Histopathology examination results were used as a reference standard to establish the diagnosis of breast cancer in the current study.

A good diagnostic method should be able to show positive results in all patients who do have the disease, and this ability can be assessed based on the sensitivity value. The sensitivity value in the current study indicated the proportion of patients with positive tumour markers among all patients who do have breast cancer (true positive or false negative results). However, a high sensitivity rate alone might not be sufficient to determine the accuracy of a tumour marker. The diagnostic method should also give negative results in all patients with benign breast tumours, an ability that is judged based on the specificity value. The specificity value in the current study indicated the proportion of patients with negative tumour markers among all patients who do have benign breast tumours (true negative or false positive results). For a test to be useful, combined sensitivity and specificity value should at least be 1.5 , and to be able to recommend the clinical use of such tests, ideally the test should be able to identify significantly more cases correctly (sensitivity $\geq 80 \%$ ) and have very high specificity $(\geq 97 \%) .{ }^{15}$

Another factor that needs to be considered in evaluating the accuracy of a tumour marker was the positive predictive value (PPV) and negative predictive value (NPV). The positive predictive value in the current study indicated the probability of patients to actually have breast cancer if the test results were positive, and the negative predictive value indicated the probability of patients to not have breast cancer if the test results were negative. It is also important to remember that the PPV or NPV are affected by disease prevalence, where PPV is positively and NPV is negatively correlated with it. Sensitivity, specificity, PPV, and NPV seem to have their advantages and disadvantages. Therefore, these four factors generally need to be evaluated together to determine the diagnostic accuracy of VEGF-C and CA 
15-3 in differentiating between patients with benign or malignant tumours. ${ }^{15}$

The current study found that combined VEGF-C and CA 15-3 has higher sensitivity and NPV in comparison with individual tumour markers, but has lower specificity and PPV. The combined examination of these two tumour markers also showed a higher level of overall accuracy. Meanwhile, VEGF-C showed an equal specificity with CA 15-3, and a higher PPV than CA 153 or combined VEGF-C and CA 15-3. Based on the these results, it is reasonable to consider the use of VEGF-C and CA 15-3 tumour markers as a screening method for patients with suspected breast cancer should the more invasive pathology examination is unavailable or undesirable, such as in patients taking anticoagulant, patients with overlying skin infections, and during pregnancy or breast-feeding. However, the overall sensitivity and specificity of the two tumour markers were still relatively too low to be recommended for actual clinical use. In addition, although the combination of the two tumour markers showed a relatively high PPV and an increased NPV, the overall NPV was still relatively too low because the number of false-negative test results was still much higher than the true-negative results. Considering the nature of malignancy, the consequences of a false positive test may involve causing unnecessary invasive or expensive follow-up procedures, while a false negative test may cause a delay in diagnosis and treatment. As a consequence, a PPV value of $\geq 90 \%$ in the current study is considered acceptable, while the very low NPV value is not.

Previous study by Zajkowska et al. also obtained a low sensitivity value, namely $65.8 \%$ for VEGF-C and $58.3 \%$ for CA 15-3. However, the study obtained a sensitivity value of $86.7 \%$ for the combination of VEGF-C and CA 15-3, indicating an increased sensitivity value when compared to individual tumour markers, ${ }^{7}$ similar to the results obtained in the current study. Specificity and NPV in the previous study generally appeared to be higher than those obtained in the current study, which were $76.0 \%$ and $52.3 \%$ for VEGF-C, $95.0 \%$ and $53.27 \%$ for CA $15-3$, and $70.0 \%$ and $72.4 \%$ for combined VEGF$\mathrm{C}$ and CA $15-3$, respectively. The study reported a higher PPV for CA $15-3$, which was $95.89 \%$, but lower for VEGF-C and the combination of VEGF-C and CA 15-3, which was $84.0 \%$ and $85.3 \%$, respectively. ${ }^{7}$

CA 15-3 has a relatively low sensitivity in stage I and II of breast cancer, and was more widely used as an early indicator of cancer recurrence, the presence of residual disease, and to evaluate remission or to predict prognosis. ${ }^{7,12,13}$ The results from a number of studies conducted over the past three decades to evaluate the use of CA 15-3 as a breast cancer screening method showed that serum CA 15-3 levels that were examined using the sandwich ELISA technique with two types of anti-CA 15-3 monoclonal antibodies (115D8 and DF3) might not be suitable when used as a single tumour marker for early detection of breast cancer because there might not be an increased CA 15-3 levels in patients with earlystage breast cancer or localized (non-invasive) breast cancer. ${ }^{1,32}$

This problem was thought to be related to the examination technique. Previous study found evidence that the use of the antibody-lectin sandwich assay to detect CA 15-3 glycosylation in serum samples could be useful for screening in early-stage or localized breast cancer. ${ }^{32}$ This examination was implemented based on the fact that CA 15-3 was a highly glycosylated protein, where changes in glycosylation were thought to be a marker of carcinogenesis, ${ }^{33}$ and that changes in CA 15-3 glycosylation have been found in breast cancer tissues. ${ }^{34}$ The antibody-lectin sandwich assay was reported to be more effective and has a significantly higher sensitivity, possibly because the ELISA technique commonly used for the examination of serum CA 15-3 levels only allows a dilution between 1:50 to $1: 100$, while the antibody-lectin sandwich assay can detect CA 15-3 in a dilution of more than 1:2000. ${ }^{32}$ However, currently the antibody-lectin sandwich assay has not been widely used to detect CA 15-3 levels, especially in Indonesia.

Previous studies have also generally investigated VEGF-C as a prognostic marker, a marker of metastases, or to predict life expectancy, ${ }^{3,14}$ and only one study evaluated the diagnostic benefits this tumour marker. ${ }^{10}$ Studies evaluating the diagnostic use of VEGF-C for breast cancer is currently limited, mainly because this protein is thought to play a greater role in lymphangiogenesis and metastasis. However, recent studies have reported the role of VEGF-C in angiogenesis and neovascularization which were considered important in early tumour development. Overexpression of VEGF$\mathrm{C}$ in breast cancer cells was found not only in invasive tumours, but also in ductal carcinoma in situ. This suggests that lymphangiogenesis may be one of the earliest events in breast carcinogenesis. ${ }^{35}$

\section{Limitations}

The current study has several limitations. Firstly, the current study did not evaluate tumour markers levels based on the stage of the disease or breast cancer types. Previous studies have reported increased tumour markers levels with increasing breast cancer stage, and increased CA 15-3 expression was more commonly found in luminal breast cancer subtypes when compared to other subtypes. ${ }^{27} \mathrm{CA} 15-3$ levels in patients with stage III or IV breast cancer appeared to be significantly higher than patients with stage I and II diseases, although VEGF-C levels did not appear to be significantly different according to cancer stages, ${ }^{7}$ and only showed a significant difference in patients with lymph node metastases. $^{36}$ Elevated CA 15-3 levels above normal values was also more common in estrogen receptor and/or progesterone receptor positive (HR+) breast cancer in comparison to hormone receptor 2 positive (HER2+) or triple negative (TP) breast cancer. ${ }^{24}$ On the other hand, HER2+ breast cancer showed a greater increase in VEGF-C levels in comparison to other types of breast cancer. ${ }^{36}$

Another limitation of the current study was that despite having generally better diagnostic value in comparison with individual tumour markers, the combination of VEGF-C and CA 15-3 was only useful for research purposes and may not be practical to use in everyday clinical practice because it is relatively difficult to determine the combined cut-off value of the two tumour markers. Previous studies have evaluated the use of ratio from two different tumour markers, such as cancer antigen 125/ carcinoembryonic antigen (CA 125/CEA) 
ratio in cases of ovarian cancer. The cut-off value based on this ratio was reported to be good enough to represent the combination of two tumour markers for diagnostic or screening purposes. ${ }^{37}$ Therefore, further studies are needed in order to evaluate the diagnostic ability of the VEGF-C/CA 15-3 ratio in breast cancer, and to determine the ratio cut-off value that can be utilized as a reference in daily clinical practice, while adjusting for menopausal status, stage of the disease or breast cancer types. The use of other potential tumor markers alone or in combination with VEGF-C and CA 15-3 should also be explored in a larger population to improve diagnostic accuracy.

\section{CONCLUSION}

Considering that the AUC indicated a moderate to good diagnostic value with relatively good sensitivity, positive predictive value, and accuracy, it is reasonable to consider the use of VEGF-C and CA 15-3 tumour markers as a screening method for patients with suspected breast cancer should the more invasive pathology examination is unavailable or undesirable. The two tumour markers may also be used to monitor the severity of breast cancer, response to therapy, or possible reccurences, even though further studies are required. Nevertheless, the use of these two tumour markers were not yet recommended for diagnostic purposes in everyday clinical practice because of the relatively low specificity and negative predictive value. VEGF-C in combination with CA 15-3 performed better as diagnostic parameter and has better accuracy as a tumour marker for breast cancer in comparison with each marker alone.

\section{REFERENCES}

1. Duffy MJ, Evoy D, McDermott EW. CA 15-3: uses and limitation as a biomarker for breast cancer. Clin Chim Acta. 2010;411(23-24):1869-74.

2. Momenimovahed Z, Salehiniya H. Epidemiological characteristics of and risk factors for breast cancer in the world. Breast Cancer (Dove Med Press). 2019;11:151-4

3. Zhang Z, Luo G, Tang H, Cheng C, Wang P. Prognostic Significance of High VEGF-C Expression for Patients with Breast Cancer: An Update MetaAnalysis. PLoS One. 2016;11(11):e0165725.

4. Sun YS, Zhao Z, Yang ZN. Risk Factors and Preventions of Breast Cancer. Int $\mathrm{J}$ Biol Sci. 2017;13(11):1387-97.

5. DeSantis CE, Fedewa SA, Goding Sauer A. Breast cancer statistics, 2015: Convergence of incidence rates between black and white women. CA Cancer J Clin. 2016; 66: 31-42.

6. Trecate G, Sinues PM, Orlandi R. Noninvasive strategies for breast cancer early detection. Future Oncol. 2016;12(11):1395-411.

7. Zajkowska M, Lubowicka E, Fiedorowicz W, Szmitkowski M, Jamiołkowski J, Ławicki S. Human Plasma Levels of VEGF-A, VEGF-C, VEGF-D, their Soluble Receptor - VEGFR-2 and Applicability of these Parameters as Tumour Markers in the Diagnostics of Breast Cancer. Pathol Oncol Res. 2019;25(4):1477-86.
8. Ouyang Y, Tsui $\mathrm{PH}, \mathrm{Wu} \mathrm{S}, \mathrm{Wu} \mathrm{W}, \mathrm{Zhou} \mathrm{Z}$. Classification of Benign and Malignant Breast Tumours Using H-Scan Ultrasound Imaging. Diagnostics (Basel). 2019;9(4):182.

9. Nigam M, Nigam B. Triple Assessment of Breast Gold Standard in Mass Screening for Breast Cancer Diagnosis. IOSR-JDMS. 2013;7(3):1-7.

10. Zajkowska M, Głażewska EK, Będkowska GE, Chorąży P, Szmitkowski M, Ławicki S. Diagnostic Power of Vascular Endothelial Growth Factor and Macrophage Colony-Stimulating Factor in Breast Cancer Patients Based on ROC Analysis. Mediators Inflamm. 2016;2016:5962946.

11. Hashim ZM. The significance of CA15-3 in breast cancer patients and its relationship to HER-2 receptor status. Int J Immunopathol Pharmacol. 2014;27(1):45-51.

12. Sunwoo HH, Suresh MR. Cancer Markers. The Immunoassay Handbook (Fourth Edition) Theory and Applications of Ligand Binding, ELISA and Related Techniques. 2013, Pages 833-56.

13. Harris L, Fritsche H, Mennel R. American Society of Clinical Oncology 2007 update of recommendations for the use of tumour markers in breast cancer. J Clin Oncol. 2007;25(33):5287-312.

14. Skobe M, Hawighorst T, Jackson DG. Induction of tumour lymphangiogenesis by VEGF-C promotes breast cancer metastasis. Nat Med. 2001;7(2):192-8.

15. van Stralen KJ, Stel VS, Reitsma JB, Dekker FW, Zoccali C, Jager KJ. Diagnostic methods I: sensitivity, specificity, and other measures of accuracy. Kidney Int. 2009 Jun;75(12):1257-1263.

16. Momenimovahed Z, Salehiniya H. Epidemiological characteristics of and risk factors for breast cancer in the world. Breast Cancer (Dove Med Press). 2019 Apr 10;11:151-164.

17. Kim Y, Yoo K-Y, Goodman MT. Differences in Incidence, Mortality and Survival of Breast Cancer by Regions and Countries in Asia and Contributing Factors. Asian Pac J Cancer Prev. 2015;16(7):28572870.

18. Assi HA, Khoury KE, Dbouk H, Khalil LE, Mouhieddine TH, El Saghir NS. Epidemiology and prognosis of breast cancer in young women. J Thorac Dis. 2013;5(Suppl 1):S2-S8.

19. Valtola R, Salven P, Heikkilä P. VEGFR-3 and its ligand VEGF-C are associated with angiogenesis in breast cancer. Am J Pathol. 1999;154(5):1381-90.

20. Thammineni KL, Thakur GK, Kaur N, Banerjee BD. Significance of MMP-9 and VEGF-C expression in North Indian women with breast cancer diagnosis. Mol Cell Biochem. 2019;457(1-2):93-103.

21. Fu S, Yun ZY, Cui MM. Cancer antigen 15-3, platelet distribution width, and fibrinogen in combination to distinguish breast cancer from benign breast disease in non-conclusive mammography patients. Oncotarget. 2017;8(40):67829-36.

22. Duffy MJ, Shering S, Sherry F, McDermott E, O'Higgins N. CA 15-3: a prognostic marker in breast cancer. Int J Biol Markers. 2000;15(4):330-33.

23. Duffy MJ, Walsh S, McDermott EW, Crown J. Biomarkers in Breast Cancer: Where Are We and Where Are We Going? Adv Clin Chem. 2015;71:123. 
24. Park S, Ahn HK, Park LC, Hwang DW, Ji JH, Maeng $\mathrm{CH}$, et al. Implications of different CA 15-3 levels according to breast cancer subtype at initial diagnosis of recurrent or metastatic breast cancer. Oncology. 2012;82(3):180-7.

25. Fu Y, Li H. Assessing Clinical Significance of Serum CA15-3 and Carcinoembryonic Antigen (CEA) Levels in Breast Cancer Patients: A Meta-Analysis. Med Sci Monit. 2016;22:3154-62.

26. Ławicki S, Będkowska GE, Szmitkowski M. VEGF, M-CSF and CA 15-3 as a new tumour marker panel in breast malignancies: a multivariate analysis with ROC curve. Growth Factors. 2013;31(3):98-105.

27. Unal I. Defining an Optimal Cut-Point Value in ROC Analysis: An Alternative Approach. Comput Math Methods Med. 2017;2017:3762651.

28. Mandrekar JN. Receiver operating characteristic curve in diagnostic test assessment. J Thorac Oncol. 2010 Sep;5(9):1315-6.

29. Power M, Fell G, Wright M. Principles for highquality, high-value testing. EvidBased Med. 2013 Feb;18(1):5-10.

30. Yavuz S, Paydas S, Disel U, Zorludemir S, Erdogan S. VEGF-C expression in breast cancer: clinical importance. Adv Ther. 2005 Jul-Aug;22(4):368-80.

31. Begum M, Karim S, Malik A, Khurshid R, Asif M, Salim, at all. CA 15-3 (Mucin-1) and physiological characteristics of breast cancer from Lahore, Pakistan. Asian Pac J Cancer Prev. 2012;13(10):5257-61.
32. Choi JW, Moon BI, Lee JW, Kim HJ, Jin Y, Kim HJ. Use of CA15-3 for screening breast cancer: An antibody-lectin sandwich assay for detecting glycosylation of CA15-3 in sera. Oncol Rep. 2018;40(1):145-54.

33. Storr SJ, Royle L, Chapman CJ, Hamid UMA, Robertson JF, Murray, et all: The O-linked glycosylation of secretory/shed MUC1 from an advanced breast cancer patient's serum. Glycobiology.2008; 18: 456-62.

34. Lavrsen K, Madsen CB, Rasch MG, Woetmann A, Odum N, Mandel, et all: Aberrantly glycosylated MUC1 is expressed on the surface of breast cancer cells and a target for antibody-dependent cell-mediated cytotoxicity. Glycoconj J.2013; 30: 227-36.

35. Raica M, Cimpean AM, Ceausu R, Ribatti D. Lymphatic microvessel density, VEGF-C, and VEGFR-3 expression in different molecular types of breast cancer. Anticancer Res. 2011;31(5):1757-64.

36. Moro F, Pasciuto T, Djokovic D, Di Legge A, Granato V, Moruzzi MC, Mancari R, Zannoni GF, Fischerova D, Franchi D, Scambia G, Testa AC. Role of CA125/CEA ratio and ultrasound parameters in identifying metastases to the ovaries in patients with multilocular and multilocular-solid ovarian masses. Ultrasound Obstet Gynecol. 2019 Jan;53(1):116-123. 